\title{
Facile Synthesis and Characterization of Biodegradable Calcium alginate nanoparticles
}

\author{
Enayah Alhashim ${ }^{1}$, Jackson Lay Jr$^{1}$ and Zoraida P Aguilar*2 \\ ${ }^{1}$ Department of Chemistry and Biochemistry, USA \\ ${ }^{2}$ Zystein, USA
}

*Corresponding author: Zoraida P Aguilar, Zystein, 76 W Sunbridge Drive, Fayetteville, AR, 72703 USA

\section{ARTICLE INFO \\ Received: 蔧 February 28, 2019 \\ Published: 㓞 March 06, 2019}

Citation: Enayah Alhashim, Jackson Lay Jr, Zoraida P Aguilar. Facile Synthesis and Characterization of Biodegradable Calcium alginate nanoparticles. Biomed J Sci \& Tech Res 15(3)-2019. BJSTR. MS.ID.002718.

Keywords: Calcium alginate nanoparticles; Biodegradable; Synthesis; Nanoparticle delivery
ABSTRACT

Alginate is one of the most abundant natural marine polysaccharides which is an attractive source of materials for the formulation of biodegradable nanoparticle delivery systems. In this study, calcium alginate nanoparticles (CALNPs) were successfully synthesized without the use of harmful organic solvents or very high temperatures. The resulting Nano emulsion of the nanoparticles showed no microbial contamination and were easily degraded when exposed to the natural environment. The empty CALNPs were bigger than those which contained either Vitamin E or Vitamin D3. The CALNPs all recorded a negative zeta potential which may serve as an indicator of long-term stability.

Abbreviations: CALNPs: Calcium Alginate Nanoparticles; CALNPs-Vit E: Calcium Alginate Nanoparticles with Vitamin E; CALNPs-Vit D3: Calcium Alginate Nanoparticles with Vitamin D3

\section{Introduction}

Alginate is one of the most abundant natural marine polysaccharides [1] which is an attractive source of materials for the formulation of biodegradable nanoparticle delivery systems. Various characteristics including biocompatibility, non-toxicity, biodegradability, and low cost make it a suitable candidate for biomedical applications [2-6]. Alginate or alginic acid, is an anionic polysaccharide that contains ma homopolymeric blocks of $(1,4)$-linked -D-mannuronate (M) unit and L-guluronate (G) [7,8]. In the form of calcium alginate gel, the calcium cations are distributed between the negative functional groups in the alginate molecules [9]. In this study, we used calcium alginate gel to synthesize calcium alginate nanoparticles (CALNPs) in a nano emulsion. The nanoparticles in the nano emulsions were characterized with optical microscope, dynamic light scattering (DLS), and TEM to establish their physical properties. The natural biodegradability of the nanoparticles was established through exposure to the natural environment that is rich in microorganism that degrades natural organic materials that are biodegradable.

\section{Materials and Methods}

Medium viscosity alginic acid sodium salt (Sigma-Aldrich) was from Dr. Ryan Tian. Poly Suga Mulse D6 and Cola Liquid DC from Colonial Chemical (TN). The following materials were obtained from Zystein (AR, USA): vitamin E (Puritan's Pride, NY), Vitamin D3 (Natural Factors, NY), soy lecithin (Amresco, OH); calcium chloride, sodium chloride, magnesium chloride, potassium biphosphate and nutrient agar (Home Science Tools, MT); HEPES buffer (Santa Cruz Biotech, Dallas, TX); olive oil (Nature's oil, $\mathrm{OH}$ ); and phenoxyethanol (Lotion Crafter, IA). All materials were used as received.

\section{Synthesis of the Calcium Alginate Nanoparticles}

A $50 \mathrm{~mL}$ aliquot of sodium alginate $(10 \mathrm{mg} / \mathrm{mL})$ was added to $100 \mathrm{~mL}$ of calcium chloride $(294 \mathrm{mg} / \mathrm{mL})$ in a beaker. The $\mathrm{pH}$ was adjusted to 3.5 using $6 \mathrm{M} \mathrm{HCl}$ or $6 \mathrm{M} \mathrm{NaOH}$ before the mixture was heated to $70{ }^{\circ} \mathrm{C}$ for $20 \mathrm{~min}$ with constant stirring. The resulting calcium alginate gel was stored at room temperature before use. In another beaker, $6 \mathrm{~mL}$ of the calcium alginate gel and $5 \mathrm{~mL}$ of soy 
lecithin $(0.023 \mathrm{~g}$ soy lecithin per mL of HEPES buffer, $\mathrm{pH}$ 6.5) were mixed. To create the particles carrying either Vitamin E or Vitamin D3, 50 to $100 \mathrm{uL}$ of either vitamin, respectively, were added before adding $0.5 \mathrm{~mL}$ of Cola Liquid DC. This was heated to $75^{\circ} \mathrm{C}$ with constant stirring until an emulsion was formed. The emulsion was cooled down to room temperature before use. The emulsion was subjected to sonication at (Branson 1510, Danbury, CT) at 10 to 30 min to reduce the particle size and create the nano emulsion.

\section{Characterization}

The emulsion was smeared thinly on a microscope glass slide that was observed under an optical microscope (American Scientific Producs, Columbus, $\mathrm{OH}$ ). The nano emulsion was diluted as necessary before characterization with TEM (JEOL USA, Peabody, MA) and DLS (Zetatrac Ultra, Microtrac Inc, Montgomeryville, PA).

\section{Microbial Contamination and Bio-degradabality Test}

The degree of microbial contamination was evaluated by plating the diluted samples $(1: 10)$ in nutrient agar plates that were incubated for $48 \mathrm{~h}$ at $37^{\circ} \mathrm{C}$. Biodegradability tests were conducted by plating the samples in nutrient agar plates that were exposed for 1-2 $\mathrm{h}$ in natural environment between 9AM to 12 noon as close as possible to the yard soil under a shade with natural air flow. All studies used DI water spread on the nutrient agar plates as control. All the microbial contamination and biodegradability tests were done in triplicate.

\section{Results and Discussion}

The emulsion that was prepared without sonication was observed under an optical microscope. The results in Figures B1, C1 and D1 showed the particles to be spherical with about 5-30 um in diameter. These were totally different from the starting sodium alginate material, (Figure 1A), which looked like strands. After sonication, shown on Figures B2,C2 and D2, the emulsion did not show any particles when observed under the optical microscope. This indicated that

a) within the resolution of the optical microscope the particles were already too small to be observed or
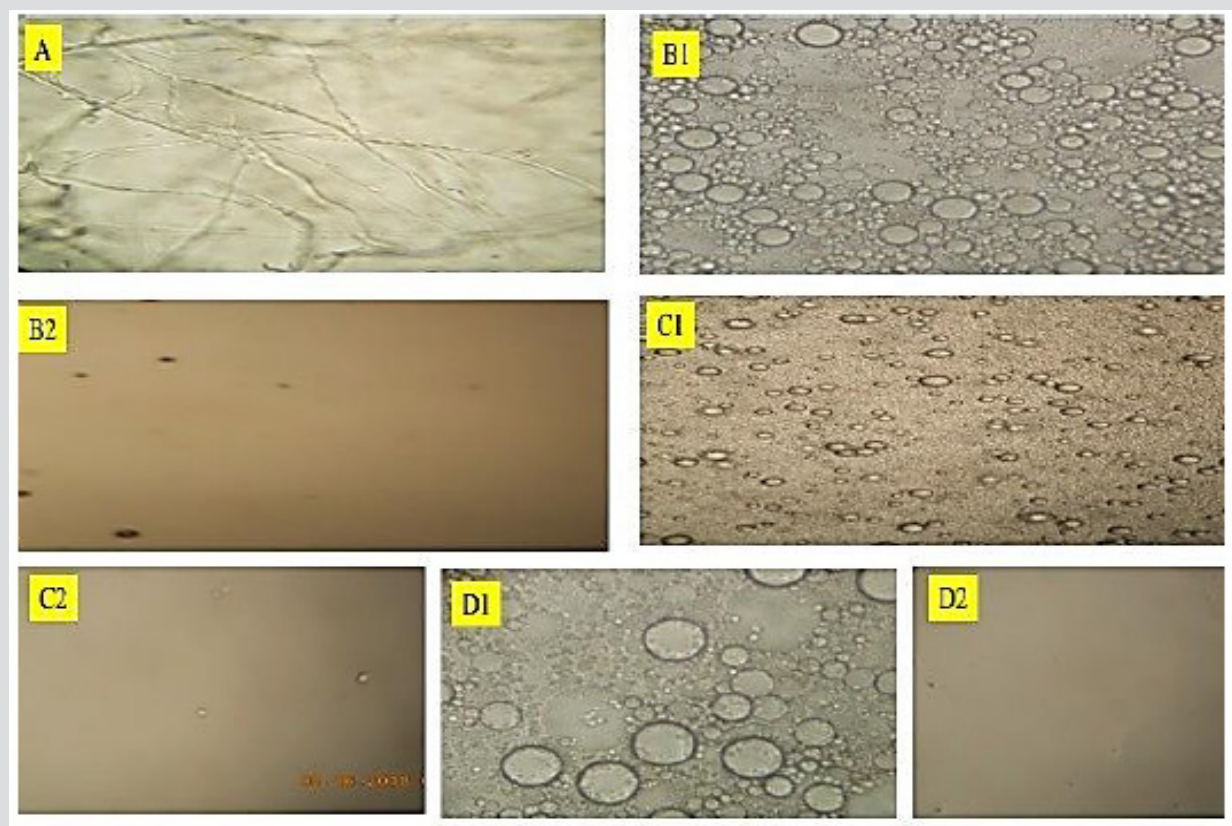

Figure 1: Results of Optical Microscope examination of the

A. sodium alginate;

B. CALNPs: calcium alginate nanoparticles;

C. CALNP-VitE: calcium alginate nanoparticles with Vitamin E; and

D. CALNP -Vit D3: calcium alginate nanoparticles with Vitamin D3. The 1 and 2 indicate before and after sonication.

b) the particles did not exist. When the sonicated emulsion was observed under TEM, the micrographs in Figure 2 showed the presence of much smaller particles which were $1185 \pm 307$ $\mathrm{nm}$ for the empty CALNPs; for CALNPs-Vit D3 and CALNPs-Vit D3 were $97 \pm 41 \mathrm{~nm}, 136 \pm 43 \mathrm{~nm}$ respectively. The results indicated that the sonication process sheared the microparticles into smaller particles in the nano size ranges. The dynamic light scattering studies were used to establish the zeta potential of the CALNPs. The negative zeta potential results (CALNPS: $-34.77 \mathrm{mV} \pm 1.63$, C ALNPs-Vit E: $-30.78 \mathrm{mV} \pm 3.07$ and CALNPs-Vit D3: $-7.01 \pm 1.66$ ) indicated that the nanoparticles may be stable over a period of time which we are currently study- 
ing. Microbial contamination studies performed over five days indicated that there was no inherent contamination in the nanoemulsions. Biodegradation studies showed that these were easily degraded within 5 days of exposure for $1-2 \mathrm{~h} /$ day to the natural environment.
A) CALNPS

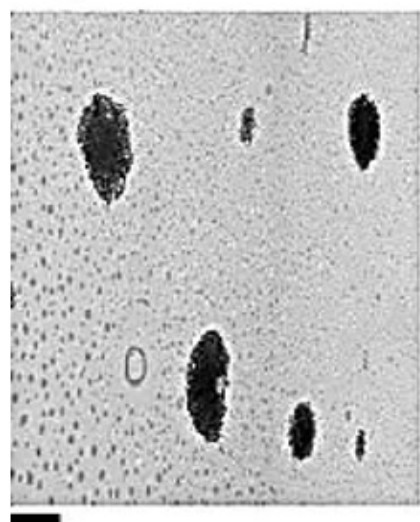

$500 \mathrm{~nm}$
B) CALNPs-Vit E

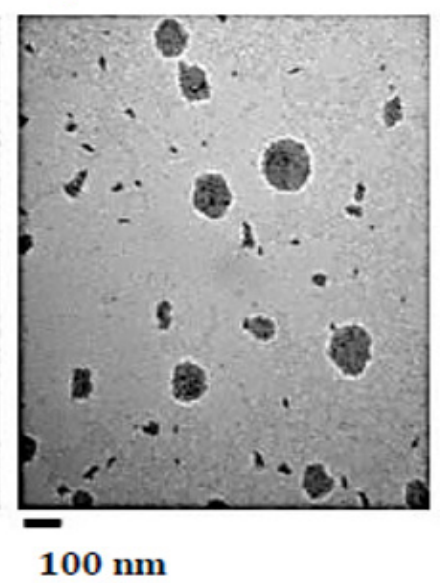

C) CALNPs-VitD3

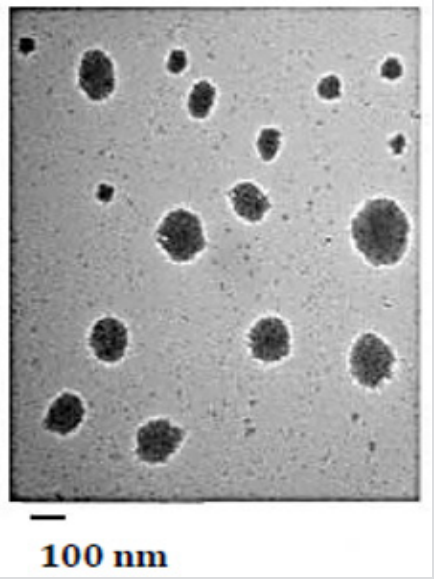

Figure 2: Results of TEM studies.

A. CALNPs: calcium alginate nanoparticles,

B. CALNPs-Vit E: calcium alginate nanoparticles with Vitamin E,

C. CALNPs-Vit D3: calcium alginate nanoparticles with Vitamin D3.

\section{Conclusion}

We have successfully synthesized calcium alginate nanoparticles without the use of harmful organic solvents or very high temperatures. The resulting nanoparticles showed no microbial contamination and were easily degraded when expose to the natural environment indicating that these would not present any environmental problems in the future because they are biodegradable. Based on the TEM results, the empty CALNPs were bigger than those which contained either Vitamin E or Vitamin D3. In our future studies, these will be tested for delivery of active ingredients for biomedical applications.

\section{Acknowledgement}

The University of Arkansas, and Zystein, sponsored all the reagents and the instruments that were used in this study. The support from the Saudi Arabian Culture Mission to the U.S. port and Dr. Israa Al Ogaidi for laboratory training. Special acknowledgement for the mentoring and guidance from Dr. Jack Lay and Dr. Zoraida Aguilar in carrying out this research.

\section{References}

1. Rinaudo M (2014) Biomaterials based on polysaccharide: alginate. TIP 17(1): 92-96
2. Lee KY, Mooney DJ (2012) Alginate: Properties and Biomedical applications. Progress in Polymer Science 37(1): 106-126.

3. Brezaniova I, Trousil J, Cernochova Z, Kral V, Hruby M, et al. (2017) Self-assembled chitosan-alginate polyplex nanoparticles containing temoporfin. Colloid and Polymer Science 295(8): 1259-1270.

4. Goswami S, Bajpai J, Bajpai AK (2014) Calcium alginate nanocarriers as possible vehicles for oral delivery of insulin. J Experimental Nanoscience 9(4): 337-356.

5. Song W, Su X, Gregory DA, Li W, Cai Z, et al. (2018) Magnetic alginate/chitosan nanoparticles for targeted delivery of Curcumin into human breast cancer cells. Nanomaterials 8: 907.

6. Straccia MC, d'Ayala GG, Romano I, Laurienzo P (2015) Alginate hydrogels coated with chitosan for wound dressing. Marine Drugs 13(5): 2890-2908.

7. Hay ID, Rehman ZU, Moradali M F, Wang Y, Rehm BHA (2013) Microbial alginate production, modification and its applications. Microbial Biotechnology 6(6): 637-650.

8. Daemi H, Barikani M (2012) Synthesis and characterization of calcium alginate nanoparticles, sodium homopolymannuronate salt and its calcium nanoparticles. Scientia Iranica 19(6): 2023-2028.

9. Fabich HT, Vogt SJ, Sherick ML, Seymour JD, Brown JR, et al. (2012) Microbial and algal alginate gelation characterized by magnetic resonance. J Biotechnol 161(3): 320-327. 


\section{ISSN: 2574-1241}

DOI: 10.26717/BJSTR.2019.15.002718

Zoraida P Aguilar. Biomed J Sci \& Tech Res

(c) (P) This work is licensed under Creative

Submission Link: https://biomedres.us/submit-manuscript.php

$\begin{array}{ll}\text { BIOMEDICAL } & \text { Assets of Publishing with us } \\ \text { RESEARCHES } & \text { Global archiving of articles } \\ \text { - Immediate, unrestricted online access }\end{array}$

